\title{
On the Physical Basis of Self-Organization
}

\author{
Mircea Sanduloviciu \\ Complex Systems Laboratory, Faculty of Physics, Alexandru Ioan Cuza University of Iasi, Iasi, Romania \\ Email: msandu@uaic.ro
}

Received January 5, 2013; revised February 6, 2013; accepted February 14, 2013

\begin{abstract}
Experiments performed with the aim to explain pattern formation in plasma devices offer, as I will show in this survey, a new insight into the mechanism by which locally matter transits spontaneously from a disordered state into an ordered one. The essential news revealed by these experiments is the identification of a population of electrons that, driven at a critical distance from thermal equilibrium, is able to act as the organizer of the emergence and the survival of a complexity starting from chaos, i.e., from electric sparks the appearance of which is controlled by deterministic chaos. Supplied at a constant rate with thermal energy extracted by electrons from plasma, the complexity survives in a dynamical state performing operations in agreement with a code directly related to electrons thermal energy distribution function. Acting as a constituent of the matter, the population of electrons intrinsically controls the emergence and the survival of the complexity. Performing operations directly related to electron's thermal energy distribution function, the complexity evolves stepwise in more advanced self-organized dynamical states, when this function is changed by an additional injection of energy. A set of nonlinear phenomena, not explainable by classical processes is involved in the mechanism by which the complexity emerges, survives and evolves. Thus, phenomena like Bose-Einstein condensation, macroscopic quantum coherence, direct and alternate Josephson effects, electron tunneling, negative differential impedance and others, potentially explain the emergence, functionality and vitality, i.e., the dynamical state of the complexity.
\end{abstract}

Keywords: Self-Organization; Physical Plasma; Complex Systems

\section{Introduction}

Self-organization is a phenomenon observed in nature and laboratory that relates the presence of order formation in complex dynamic systems, i.e., systems whose state at one moment of time is a function of the state at the previous moment of time and of the energy input. Inspired by experimental results [1-3], the science of complexity was grounded on principles as "order from noise", (Heinz von Förster), "order through fluctuations", (Ilya Prigogine) and the "slaving principle" (Herman Haken). In spite of intense investigations, the science of self-organization as a whole is still little more than a collection of examples, methods and metaphors for modeling its emergence and behaviors [4]. Thus, at present, a phenomenological model and implicitly an integrated theoretical explanation of self-organization, describable in terms of mathematical equations, misses.

Questions as the followings remain to day not answered [5]:

- Which perturbations and parameters sensitively control the pattern formation?

- What are the mechanisms by which small effects govern the dynamics of pattern formation?

- What are the interrelations between physics at differ- ent length scales in pattern-forming systems?

- When and how do atomic-scale mechanisms control the macroscopic phenomena?

Answers, potentially able to offer solutions to the aforelisted questions, are offered by plasma experiments the aim of which was to explain the emergence and behaviors of a coherent relative stable luminous gaseous body known as fireball [6]. A synthesis of these experimental results and of the used references is given in [7].

The investigation was performed using a diode which conductor was argon plasma. Minuscule electric sparks produced at the surface of the anode, under the circumstances that the electrons located in the plasma are driven at a critical distance from thermal equilibrium by the applied electric field, initiate the emergence of the fireball. The sparks, whose appearance is controlled by deterministic chaos, are minuscule well-located hot nonequilibrium dusty plasmas that result after the vaporization of the anode material from the top of protuberances where the current is concentrated. The essential news revealed by these experiments consist in the identification of the mechanism by which a population of electrons driven at a critical distance from thermal equilibrium, acting in a collective way, are able to create order starting from chaos, i.e., from the minuscule electric sparks 
afore-mentioned. Thus, when the initial temperature of the non-equilibrium dusty plasma i.e., of the spark, surpasses a critical value, instead of evolving into a state of thermal equilibrium (as required by the second law of thermodynamics) it quasi-instantaneously evolves into a spatio-temporal ordered state in the form of the fireball. The investigation of the fireball performed so far, revealed the following phenomena [7]: 1) The coherence and relative stability of the fireball is related to the presence of a nearly spherical electric double layer at its border; 2) The double layer sustains a positive anode fall with the same order of magnitude as the ionization potential of the background gas and a negative anode fall having the order of magnitude of some volts; 3) The kinetic temperature of the electrons inside the fireball is three times greater than that of the surrounding electrons; 4) The density of the current that flows to the anode in the point where the fireball is attached is two-times greater than the mean anode current density; 5) The light emitted by the fireball is not related to the thermal excitations of the atoms; 6) Negative conductance oscillations are stimulated by the fireball.

For initiating the emergence of the fireball starting from an electric spark, the plasma located in the diode was driven by the applied electric field at a critical distance from thermal equilibrium. Being accelerated in this electric field, the electrons produce atom ionizations, and, implicitly excitations. Consequently, by accumulation of opposite space charges at the distance from the anode where the ionization and excitation cross-section functions suddenly increase, the symmetry of the particle distribution in the plasma is broken. The evolution of the spark into the fireball depends on the initial temperature of the dusty plasma located in it, i.e., two routes of evolution are possible. So, a bifurcation takes place. Once emerged, the space-charge arrangement of the fireball emphasizes long-range order. Since phenomena as nonequilibrium, symmetry breaking, bifurcation and longrange order represent key processes of self-organization [8] the emergence of the fireball was considered, to the best of my knowledge for the first time, to be a typical example of self-organization (see the ref. in [7]).

Self-organization, as today defined, is that phenomenon at the origin of which no external forces can be identified to act as organizer [9]. In this context, the novelty revealed by the emergence of the fireball is that, such an organizer exists in the form of a population of electrons driven at a critical distance from thermal equilibrium by an external constraint [7]. In the plasma diode, the external constraint is the electric field sustained by the dc power source. Emerging quasi-instantaneously through a bifurcation-like instability (i.e., a phenomenon that usually serves as memory mark) the fireball has encoded (during its emergence) information directly related to the shape of electrons thermal energy distribution function. So, the fireball posses a so-called electric memory, which enables it to survive in a dynamical state performing the operations "learned" during its emergence [7]. The mechanism, by which this memory works, is based on the capacity of electrons to store information in the patterns, i.e., the electric skeleton of the fireball, by converting thermal energy into electric field energy.

Once emerged, the fireball survives in a dynamical state by a mechanism, supplied at a constant rate with thermal energy extracted by the electrons from the plasma. Taking into consideration that the population of electrons is itself a constituent of the plasma (i.e., of the fourth aggregation state of the matter) their capacity to create ordered patterns starting from chaos (i.e., from the minuscule electric sparks) is an event by which matter prove its ability to self-organize. On the other hand, owing to the fact that for working as an organizer the population of electrons must be driven at a critical distance from thermal equilibrium, the self-organization process by which the fireball emerges and survives requires work done by the dc power source that act as an external constraint. So, there exists a strong interdependence between the environmental conditions - the plasma driven at a critical distance from thermal equilibrium - and the emergence and survival of the fireball. So, as experimentally proved [7], changing the thermal energy distribution function of the electrons by increasing the dc voltage of the power source the fireball evolves into more advanced dynamical states i.e., self-organized states. The evolution takes place stepwise each of the dynamical states involving a bifurcation-like instability that serve as a memory marks. Phenomena by which the fireball emerges and survives in a dynamical state, remind of those revealed when the matter is in the so-called super-states. Such states involve Bose-Einstein condensation, macroscopic quantum coherence, electron tunneling, Josephson effects but also of other unusual ones.

In the following, the presence of the above briefly mentioned phenomena are illustrated through experimental results obtained by investigating the emergence and behaviors of the fireball in a plasma diode, known as low-voltage arc. The novelty revealed by these investigations is represented by the capacity of the fireball to evolve, transiting through a set of three bifurcation-like instabilities, each of them serving as memory mark, towards a free-floating state. Known as ball-of-fire, the survival of the free-floating fireball involves an advanced dynamical state that implies a rhythmic exchange of matter and energy with the surrounding plasma.

\section{Self-Organization Phenomena in the Low Voltage Arc}

Briefly speaking, the low-voltage arc contains a tungsten 
cathode heated at the temperature for which positive ions are generated by contact ionization and free electrons are produced by thermo-emission and a tungsten plate that acts as anode. The electrodes are located in a chamber that contains vaporized cesium as background gas i.e., atoms characterized by low ionization potential. The plasma created by the cathode reaches by diffusion the anode. A dc power supply PS connected to the anode through a load resistor sustains an electric field between the electrodes the intensity of which can be varied in a controllable way. Subjected to this electric field, the plasma caries current i.e., it is driven away from thermal equilibrium at a distance that depends on the PS-voltage. By gradually increasing the PS-voltage, a typical static $I(V)$ characteristic (as shown in Figure 1) is obtained.

This characteristic contains branches whose slopes are directly related to the collective effects produced by the elastic and inelastic collisions of the accelerated electrons with the atoms. Beginning to gradually increase the PSvoltage, firstly the branch marked a-b is run through by the current $I$. In this interval of the PS-voltage, the current $I$ increases in proportion with the potential $V$ of the anode i.e., Ohm's law is valid. The slopes of the $I(V)$ characteristic change when, by the accumulation of the electrons that lose their kinetic energy after atom ionizations in front of the anode, a barrier for $I$ appears (branch b-c). A luminous sheath attached to the anode surface resulting after the transition of the excited atoms in the ground state proves this statement. When further increasing the PS-voltage, the potential of the anode reaches the value for which the first atom ionization takes place.

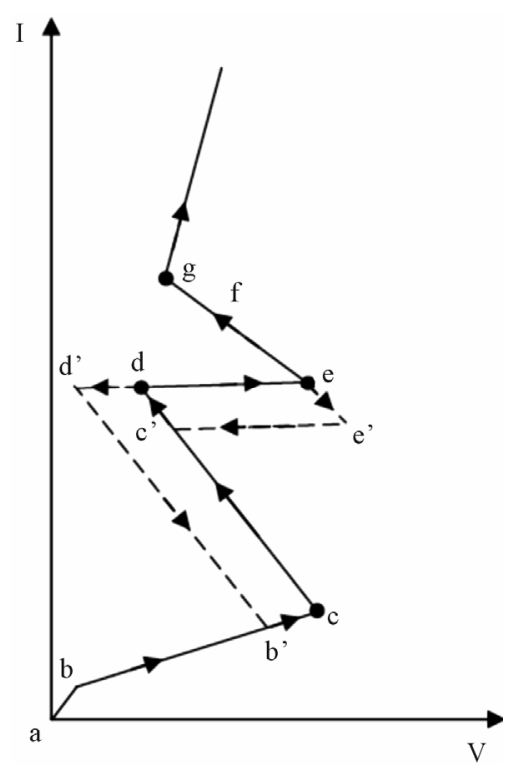

Figure 1. Typical static $I(V)$ characteristic of a low voltage arc (the values of the anode potential for which the ball-offire in stable state and respectively in pulsating state quasiinstantaneously emerges are marked by $f$ and $g$ ).
Their appearance initiates the emergence of a planar electric double layer owing two essential different causes [7]. The first one is the mobility differences between the positive ions and the electrons, while the second one is the differences in their thermal diffusivity. The first cause involves the accumulation of positive ions at the distance from the anode where the ionization cross-section function suddenly increases. Located in the next vicinity of the region where electrons accumulate after atom excitations, a planar double layer is self-assembling owing to the electrostatic forces that act as long-range correlation forces between the two adjacent net opposite space charges. Its existence implies the collection by the anode of the electrons at a higher rate than the rate by which the positive ions leave this region. The second cause determines an additional localization of positive ions at the positive side of the double layer by a mechanism identified by us [7], namely, the capacity of electrons to produce, concomitant with atom ionizations, an increase of their kinetic temperature. Previously well established by probe measurements [10] this phenomenon was considered by the authors not to be explainable by classical processes.

Owing to the quicker thermal diffusivity of the electrons with respect to that of the positive ions, an excess of positive ions remains located at the positive side of the double layer. We explained their localization starting from the following hypotheses [7]: 1) The atom ionizations are produced by electrons whose kinetic energy equals or surpasses (with an amount equal to their thermal energy) the ionization threshold energy; 2) During atom ionizations, the thermal energy achieved by the electrons in the plasma, becomes free, so that, acting in a collective way, an increase of their kinetic temperature takes place. Evidently, owing to the randomness of electrons thermal motion, only a part of them contribute to the increase of their kinetic temperature. The momentum of these electrons must be so oriented that it increases their kinetic energy when the atom ionization processes take place.

Accepting the afore-mentioned hypotheses, the appearance of the branch marked c-d in Figure 1, whose slope is negative, becomes explainable by taking into account the presence of minuscule electric sparks at the surface of the anode, the appearance of which is controlled by deterministic chaos. As afore-mentioned, the sparks are minuscule well-located hot dusty non-equilibrium plasmas that result after the vaporization of the anode material from the tops of the protuberances present at the surface if the anode where the current is concentrated. The electric sparks determine the growth of the ionization rate at the positive side of the double layer increasing in this way the kinetic temperature of the electrons. So, owing to the difference in thermal diffusivity between the posi- 
tive ions and the electrons, the positive ions remain in excess, located at the positive side of the double layer. The electrons, initially located in the negative side of the double layer after atom excitations, electro-statically couple, at one moment of their thermal motion, with these positive ions. In this way, elementary electric dipoles self-assembly by a mechanism related to the electrons thermal motion. Subjected to the action of the electric field sustained by the PS, the dipoles form a planar structure of dipoles (PSD) located inside the double layer. These dipoles are so oriented that their positive poles remain located in the positive side of the double layer. The constancy of the dipoles concentration is realized in a dynamical state that involves a balance between the number of dipoles that self-assemble at one moment and the number of the dipoles that disintegrate in the following moment by recombination of their positive ions with the free electrons. The very short but finite time-span that exists between these two phenomena enable the PSD to sustain a proper drop of potential. As the electrons are accelerated in the electric field sustained by the PS but also in the well-located potential drop of the PSD, it results that a part of their kinetic energy has its origin in their acceleration in the potential drop sustained by the PSD. Driven by work done by the PS, at a critical distance from thermal equilibrium, the descendant branch of electrons thermal energy distribution function has slopes that depends on the relationship between the rate by which their number increases when their thermal energy decreases at a constant rate [7]. Accelerated in the potential drop of the PSD the electrons produce an increase of the ionization rate and, implicitly, an increase of their kinetic temperature at the positive side of the PSD. Owing to the increase of electrons kinetic temperature, the concentration of positive ions increases at the positive side of the PSD by a mechanism related to the difference between the thermal diffusivity of positive ions and electrons. Consequently, through the electrostatic coupling of the electrons located at the negative side of the double layer, new dipoles are incorporated in the PSD. As a result its potential drop increases again. Continuing to gradually increase the PS-voltage, this phenomenon repeats [7]. So, by successive acceleration in the potential drop of the PSD of electrons the number of which increases when their thermal energy decreases the evolution of the double layer and of the PSD takes place simultaneously [7]. Each of the dipoles sequester, during the self-assemblage process, an amount of electric field energy that originates in a mechanism by which thermal energy extracted by the electrons from the plasma is directly converted into electric field energy. This means that the electric field energy stored in each dipole has its actual origin in thermal energy extracted by a group of electrons the number of which is, in average, the same. Owing to the fact that the thermal motion of the electrons is involved in initiating the electrostatic coupling of the electrons with the positive ions, this phenomenon takes place lossless, i.e., no additional work done by the PS is involved in producing this phenomenon.

The evolution of the PSD i.e., the incorporation of new dipoles, involves work done by: 1) The PS-for accelerating the electrons at energies that are sufficiently high to produce atom ionizations, and implicitly excitations, for driving the plasma at a critical distance from thermal equilibrium, for compensating the thermal energy dissipated by the resistors present in the circuit and for creating the minuscule electric sparks; 2) The plasma source i.e., the cathode that, heated by a proper power source, acts independently from the PS, replaces the thermal energy extracted by the electrons from the plasma. By directly converting the thermal energy into electric field energy the electrons sustain the potential drop of the PDS using the power delivered by the plasma source. This takes place in a dynamical state that, as afore-shown, involves a balance between the rates by which new dipoles self-assembly at one moment and the rate by which, in the following moment, the dipoles disintegrate by recombination of their positive ions with the free electrons. This means that, the existence of the PSD involves the emission of coherent radiation. The evolution of the PSD concomitant with the gradual increase of the PS-voltage, takes place through a succession of dynamical states. The increase of the ionization rate during the evolution of the PSD determines the decrease of the internal resistance of the low-voltage arc. Consequently, due to the presence of the load resistor, the static $I(V)$ characteristic shows the presence of a negative conductance (branch d-e in Figure 1).

The evolution of the PSD, involving the collection of electrons that produced and resulted after ionizations by the anode, ends when the potential at its positive side equals the potential of the anode. In this moment, instability starts, after which the PSD spontaneously transits into an equilibrium state, known as anode glow [11]. Its appearance is related to the fact that the electrostatic forces that act as correlation forces between the two adjacent opposite net space charges of the PSD become dominant with respect to the forces that act on it due the presence of the electric field sustained by the PS. As a result, after the transition into a state characterized by a minimal value of the potential energy, the anode glow attached to the anode surface emerges spontaneously. The photograph shown in Figure 2(a) proves its presence in the low-voltage arc.

This state of the low voltage arc is, as experimentally proved, unstable with respect to very small variations of the anode potential $V$. These variations appear randomly owing to the presence of the electric sparks at the surface 


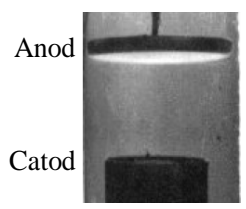

(a)

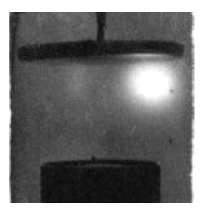

(b)

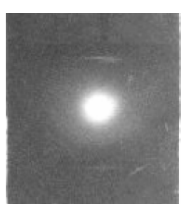

(c)

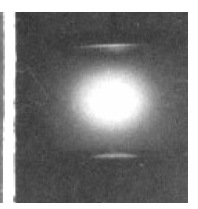

(d)
Figure 2. Photographs of the pattern formation processes in the low voltage arc: anode glow (a), fireball (b), stable ballof-fire (c) pulsating ball-of-fire (d).

of the anode. So, when the initial temperature of the well-located dusty plasma, i.e., of the spark, surpasses a critical value, the fireball quasi-instantaneously emerges through a bifurcation-like instability [7]. The fireball appears (as shown in Figure 2(b)) attached to the point where the well-located dusty plasma has initiated its emergence. The evolution of the well-located dusty plasma into the fireball is associated with the presence of a very sharp spike in the current collected by the anode [7]. A so-called current overshoot [12] that accompanies the quasi-instantaneous emergence of the fireball also proves the presence of this phenomenon. Directly related to the abrupt increase of the ionization rate, this spike-mode increase of the current takes place under constant anode potential.

Consequently, the increase of the ionization rate is not related to the increase of electrons kinetic energy. So, the single possibility to explain this spike-mode increase of the current is to consider that the number of electrons that produce the ionization increases in the same manner. The mechanism able to explain such a spike-mode increase of the ionization rate under constant anode potential implies as revealed by us [7] the presence of a population of electrons whose descendant branch of their thermal energy distribution function has slopes nearly perpendicular to the thermal energy axis. So, directly related to the spike-mode increase of the current, the emergence of the fireball takes place quasi-instantaneously starting from the well-located hot non-equilibrium dusty plasma. Due to the difference in thermal diffusivity between the positive ions and the electrons inside the well-located dusty plasma, a nucleus enriched in positive ions emerges [7]. The nucleus, that has its origin in the anode material vaporization, has a potential that surpasses the potential of the anode. Acting as a gas anode, the nucleus accelerates the electrons extracted from the surrounding plasma. The electrons that produce ionizations and excitations of the atoms, initiate the formation of a nearly spherical electric double layer by a mechanism similar to that which stays at the origin of the anode glow. The first new ionizations at its positive side are produced by accelerating (in the potential drop sustained by this spherical double layer) the group of electrons extracted from the surrounding plasma with maximum thermal energy.
Simultaneously with the ionizations produced by this group of electrons, the electrons kinetic temperature increases. Consequently, positive ions are accumulating in excess at this side owing to electrons higher thermal diffusivity with respect to that of the positive ions.

Because the positive ions are located in the next vicinity of the region where the electrons accumulated after atom excitations, there exists the chance for the electrons to electro-statically couple, at one moment of their thermal motion, with these positive ions. So, a nearly spherical structure of dipoles (NSSD) that borders the positive nucleus emerges. A dynamical state that involves a balance between the rates at which, at one moment, new dipoles self-assembly and the rates by which in the following moment the dipoles disintegrate by recombination of their positive ions with the free electrons, ensures the survival of the NSSD. Similarly to the dynamical state of the PSD and, implicitly, of the anode glow, the infinitesimal time span that exists between these two phenomena explains the survival of the NSSD. Surrounded by a population of electrons additionally accelerated in the anode glow, the population of electrons that surrounds the positive nucleus is driven at that critical distance from thermal equilibrium for which, as experimentally proved, the evolution of the positive nucleus into the fireball takes place [7]. The evolution involving a quasi-instantaneous increase of the intensity of the current and, implicitly, of the ionization rate produced under constant anode potential, reveal that the descendant branch of the electrons thermal energy distribution function has slopes nearly perpendicular to the thermal energy axis. Under such initial conditions the positive feedback mechanism described in [7] explains the evolution of the positive nucleus into the fireball. This positive feedback mechanism involves a self-enhancement of the number of positive ions at the positive side of the NSSD by a mechanism during of which the thermal energy extracted by the electrons from the plasma act as a catalyst by which the concentration of positive ions at its positive side quasi-instantaneously increases. So, the evolution of the positive nucleus into the fireball involving a quasiinstantaneous increase of the current and, implicitly, of the ionization rate produced under constant anode potential, is an event that emphasize the presence of a descendant branch of the electrons thermal energy distribution function the slopes of which are nearly perpendicular to the thermal energy axis [7].

Accelerated in the potential drop of the NSSD, the group of electrons the thermal energy of which is maximally produces the first ionizations at its positive side. Since concomitant with the ionizations the electrons increase also their kinetic temperature, it results that positive ions are additionally located at the positive side of the NSSD owing to the difference in thermal diffusivity 
between positive ions and electrons. With these positive ions, the electrons located at the negative side of the nearly spherical double layer electrostatic, couple. In this way, new dipoles are incorporated in the NSSD and, implicitly, its potential drop increases. Additionally accelerated in this increased drop of potential, the following group of electrons (whose thermal energy is smaller but their number is greater) produces the next ionizations at the positive side of the NSSD. Concomitantly with these ionizations, the kinetic temperature of the electrons again increases. So, new positive ions are incorporated in the NSSD. This phenomenon repeats with a speed that depends on the slopes of the descendant branches of electrons thermal energy distribution function. Related to the very quick increase in the current $I$ i.e., to the current overshoot, the growth of the ionization rate takes place quasi-instantaneously. The kinetic temperature of electrons, increasing in proportion to the ionization rate, the evolution of the NSSD i.e., the incorporation of dipoles in its conformational, takes place quasi-instantaneously. As a consequence, the NSSD expands in the same way up to the moment when the ionization rate (the value of which is limited by the concentration of the atoms) balances the rate by which the dipoles disintegrate by recombination of their positive ions with the free electrons. So, the fireball has a size that depends on the pressure of the background gas. The evolution of the well-located dusty plasma into the fireball, being initiated from a positive nucleus the potential of which was greater than the anode potential, it results that the positive side of the NSSD surpasses the potential of the anode. Consequently, for reaching the anode, the electrons must run through a decelerating electric field i.e., in a direction where electrons don't usually run. A tunneling like phenomenon of the electrons takes place. Located in the current carrying plasma, opposite net space charges accumulate at the two sides of the NSSD. The presence of a nearly spherical double layer [11] at the border of the fireball becomes in this way explainable. Formed by an arrangement of dipoles, the self-consistence of which is ensured by dipoledipole interactions i.e., by van der Waals and London forces, the NSSD is so thin that its presence can not be displayed by usual electric probe measurements. Similarly to the PSD, the existence of which is proved by the emergence of the anode glow, the existence of the NSSD is proved by the emergence of the fireball.

Involving a mechanism by which electrons electrostatically couple with positive ions, the emergence of the NSSD implies a very quick cooling process. So, a BoseEinstein-like condensation can be associated to the quasiinstantaneously emergence of the NSSD and, implicitly, to the emergence of the fireball. Sustained by a mechanism through which electrons directly convert thermal energy extracted from the plasma into electric field en- ergy the potential at its positive side of the NSSD is greater than the potential of the anode. Consequently, the NSSD propels electrons into the nucleus of the fireball and partly to the anode. Producing in this way direct current the NSSD behaves like a direct current Josephson junction. Macroscopic quantum coherence like that stipulated by Fröhlich apparently takes place during the emergence of the NSSD by considering the population of electrons driven at a critical distance from thermal equilibrium as a "hot bath" and the NSSD as a "giant dipole" [13]. The NSSD, being a self-consistent structure of elementary dipoles, shows a behavior similar to that revealed by small systems. Thus, the functionality of the NSSD in a dynamical state involves the constant rate transport of thermal energy extracted by the electrons from a cold reservoir, namely, the surrounding plasma, into a hot reservoir located at its positive side. So, working in a reversed mode with respect to the usual thermal machines, the NSSD produces current. Involving a balance between the rate by which new dipoles self-assemble at a moment and the rate by which these dipoles disintegrate in the following moment, the survival of the NSSD and, implicitly, of the fireball implies the emission of monochromatic radiation by disintegrations of the elementary dipoles. This emission takes place independently from the emission of light related to the transition of the excited atom (located at the negative side of the fireball into the ground state. Surviving in this way, the NSSD and, implicitly, the fireball behaves like a dissipative structure [8]. However, owing to the fact that the radiation emitted has its origins in the direct conversion of thermal energy extracted by the electrons from the plasma, and that it appears entirely in the surrounding also as thermal energy, it results that the entropy of the system consisting in the plasma source and the surroundings is not changed. This is a hint that the quasi- instantaneous emergence of the NSSD takes place in a time span in which the second law of thermodynamics is not defied but stalled. In other words, the second low of thermodynamics does not have the time to work during the quasiinstantaneously emergence of the NSSD.

The above described phenomena concerning the emergence and survival of the fireball in the low voltage arc present strong similarities with that observed in a diode the conductor of which is an argon plasma [7]. Thus, adjusting the PS voltage so that the anode potential has the value corresponding to the point marked c in Figure $\mathbf{1}$ the low voltage arc generates free-running oscillations related to an S-shaped negative differential resistance (branches b-c-d-e in Figure 1). During the oscillations, the current $I$ runs through the branches of the hysteretic cycle marked c-d-d'-b'-c in Figure 1. The origin of this hysteretic behavior emphasized by $I$ is the presence of the fireball [7]. Thus, adjusting the potential $V$ at the 
value corresponding to the point marked c in Figure 1, the fireball quasi-instantaneously emerges through a bifurcation like instability. Owing to the mechanical plotter used for inscribing the static $I(V)$ characteristics of the low-voltage arc and the shortness (some $n s$ ) of the spikemode increase of $I$, this phenomenon is not registered by the plotter. So, the informational content offered by plotting the static $I(V)$-characteristic misses the information related to the quasi-instantaneously emergence of the NSSD and, implicitly, of the fireball.

Once emerged, the fireball survives when decreasing the PS voltage the potential of the anode varies from the value corresponding to the point marked $\mathrm{d}$ to that marked d'. This behavior of the fireball reveals that once emerged, it possesses a proper memory. Related to a hysteretic phenomenon this memory is called electric memory. Supplied with thermal energy extracted by the electrons from the plasma the NSSD performs, in the afore-mentioned interval of the anode potential, all operations needed to sustain the potential at its positive side greater than the potential of the anode. So, the NSSD propelling electrons to the anode, the high conductive state of the low-voltage arc is sustained in this interval of the anode potential. When owing to the presence of the load resistor this potential decreases, reaching the value marked d', the fireball disintegrates. Consequently, the low-voltage arc spontaneously transits into the low conductive state (branch b'-b). Consequently, by work done, the PS the potential of the anode increases again at the value marked $\mathrm{d}$ for which, a new electric spark initiates the emergence of a new fireball. So, by successive emergence and disintegration of fireballs, negative conductance oscillations related to an S-shaped negative differential resistance are generated by the low voltage arc.

When the PS voltage is increased so that the potential $V$ surpasses the value corresponding to the point marked d in Figure 1, a new critical value of the anode potential is reached, for which another type of free-running oscillations are generated by the low-voltage arc. These are related to an $\mathrm{N}$-shaped negative differential resistance that works when the branch d-e is run trough by I. Like a diode, the conductor of which was argon, the transition between the aforementioned two kinds of oscillations involves a period doubling phenomenon accompanied by amplitude halving ones [7]. In Figure 3, photographs that prove this phenomenon for the low voltage arc are presented.

The potential of the anode corresponding to the point marked e in Figure 1 corresponds to the value for which the slopes of the static $I(V)$ characteristic become again negative So, continuing to gradually increase the PSvoltage, a critical value of the anode potential is reached for which, spontaneously, the fireball detaches from the surface of the anode. Dubbed as ball-of-fire its emergence

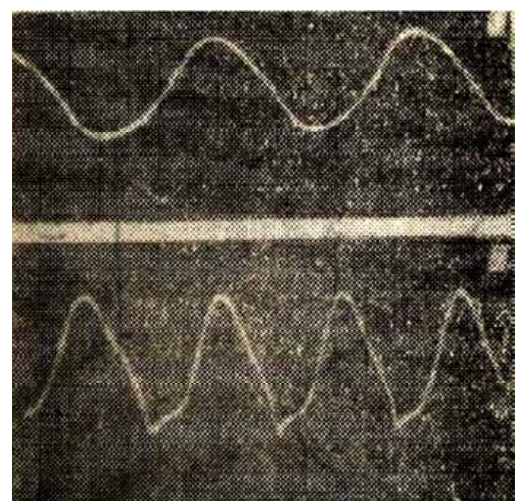

Figure 3. Two kinds of oscillations related to a period doubling phenomenon.

and behaviors were intensively investigated but a general accepted explanation concerning its actual origins is missed at present. This phenomenon becomes explainable considering that, by increasing the PS-voltage, the population of electrons, initially located between the anode glow and the anode, extends its presence in the entire volume between the cathode and the anode. So, the population of electrons that surrounds the positive nucleus has a descendant branch of their thermal energy distribution function the slopes of which are nearly perpendicularly to the thermal energy axis. Under such conditions, the presence of a spherical structure of dipoles at the border of the nucleus exists. The electrons accelerated to the positive nucleus from all sides are able to directly convert thermal energy into electric field energy. In this way, a spherical structure of dipoles emerges at the border of the nucleus. This structure, involving direct conversion of thermal energy extracted by the electrons from the surrounding plasma, sustains the potential at its positive side greater than the potential of the anode. Accelerated in the potential of this spherical structure of dipoles, a part of electrons, namely that traverses it without inelastic collisions, reaches the nucleus. Producing here ionizations of the atoms and, implicitly, an increase of their kinetic temperature, a positive charging process of the ball-of-fire nucleus takes place owing to the difference in thermal diffusivity between positive ions and electrons. In this way the nucleus of the ball-of-fire is positively charged. A phenomenon not explained up yet namely, the ability of the ball-of-fire to sustain the potential of its nucleus greater than the potential of the anode becomes explainable in this way. By this mechanism, the ball-of-fire survives also in the absence of the externally connected PS [7]. So, connecting the anode through a resistor directly to the cathode the low-voltage arc will work as a socalled thermionic converter, i.e., a diode able to convert directly thermal energy into current.

The ball-of-fire spontaneously transits into a more advanced state of dynamical organization by continuing to 
increase the PS-voltage. In this state, spherical dipolar structures periodically detach and reform from/at its border. These spherical structures departing from the nucleus like a soliton disintegrate at a certain distance. The electrons initially bounded at the dipolar structures become in this way free. Subjected to the electric field sustained by the positive nucleus, the electrons reach the region where a new spherical dipolar structure appeared. Accelerated in this structure, the electrons produce new ionizations and, implicitly, an increase in the electrons kinetic temperature at its positive side. So, after the new incorporation of dipoles in the spherical structure of dipoles its peeling off process is again initiated. In this new dynamical state the ball-of-fires survives performing a rhythmic exchange of matter and energy with the surroundings.

\section{Conclusions and Perspectives}

Governed by a conformational dynamic based on the capacity of electrons to convert directly thermal energy into electric field energy stored in ordered arrangements of dipoles the survival of the fireball involves the action of quantum mechanical forces as van der Waals and London forces. Bose-Einstein-like condensation, macroscopic quantum coherence and quantum computation occur as collective effects due to the afore-mentioned capacity of electrons.

Originating from deterministic chaos the emergence of the ball-of-fire emphasizes characteristics of a complexity whose functionality and vitality justify its consideration [7] as an example by which matter creates itself order starting from disorder. So, a paradigmatic shift in conceptually understanding self-organization is possible in the absence of principles.

The informational content offered by plasma experiments described in this survey potentially offers, as previously shown answers able to elucidate many of the enigmas revealed by the nature. Originated in an event that appears randomly, namely, the well-located dusty plasma created by the vaporization of the matter in a point where a linear lightning strikes the surface of the earth, the appearance of the ball lightning becomes explainable considering a phenomenology similar to that at the origin of the ball-of-fire [14]. Accepting that at the surface of the earl (abiotic) earth, in absence of water and organic matter, locally (for ex. in craters of volcanoes) plasma like that in the low-voltage arc existed the presence of a special kind of life, that preceded the appearance of organic life, recently dubbed inorganic life [15] seems to be possible [16].

A phenomenology as that at the origin of the ball-of fire was considered by us to be able to offer a new insight into the mechanism by which the nature creates the first form of organic life [7]. We start from the hypotheses that minuscule electric sparks created balls-of-fire with sizes of micro/nanometers at the surface of the earl earth when water and organic matter appeared. Under such premises the emerged electric skeleton, i.e., the spherical structure of elementary dipoles that border the minuscule ball-of-fire potentially acts as a mould on which proteins and protein assembles attach $[7,17]$. Once emerged, their conformational dynamic is governed by quantum mechanical forces as afore mentioned. Quantum effects exhausted at scales larger than individual atoms or sub-atomic particles apparently explain the dynamical state of the minuscule ball of fires. Playing functional roles at mesoscopic scales, the dipoles perform operations that remind that of living (biological) cells. This involves a mechanism by which the electrons originating from a population driven at a critical distance from thermal equilibrium by an external constraint act as organizer.

Having at its genuine origin fluctuations and noise generated by minuscule electric sparks the emergence of ordered structure of elementary dipoles as described in this survey potentially reveals how nature created complexities the dynamical state of which is governed by quantum mechanical forces. Accepting the hypothesis that the so-called microtubules of hollow cylindrical polymers [18] actually are elementary dipoles the consciousness and, implicitly, the essence of life could be potentially explained. This explanation is based on the capacity of a population of electrons driven at a critical distance of thermal equilibrium by an external constraint to act as an organizer and sustainer of a dynamical state that involves the action of quantum mechanical forces. The electrodynamic activity of the contemporary living cells and of assembles of living cells become potentially explainable in this way.

\section{REFERENCES}

[1] H. Bénard, "Le Tourbillons Cellulaires Dans un Nape Liquide," Revue Generale des Sciences Pures et Appliquees, Vol. 11, 1900, pp. 1261-1271, 1309-1328.

[2] A. M. Zhapotinsky, "Periodic Liquid Phase Reaction," Proceedings of the USSR Academy of Sciences, Vol. 157, 1964, pp. 392-395.

[3] H. Haken, "Synergetics. Introduction and Advanced Topics,” Springer, Berlin, 2004.

[4] F. Heyligen, "Complexity and Self-Organization,” Taylor \& Francis, New York, 2008.

[5] J. P. Gollub and J. S. Langer, "Pattern Formation in Nonequilibrium Physics,” Reviews of Modern Physics, Vol. 71, No. 2, 1999, pp. S398-S403.

doi:10.1103/RevModPhys.71.S396

[6] B. Song, N. D’Angelo and R. N. Merlino, "On Anode Spots, Double Layers and Plasma Contactors," Journal of 
Physics D: Applied Physics, Vol. 24, No. 10, 1991, pp. 1789-1795. doi:10.1088/0022-3727/24/10/012

[7] E. Lozneanu and M. Sanduloviciu, "Phenomenology of the Ball Lightning and Its Relevance for Other Enigmas of the Nature,” Nova Science Publisher Inc., New York, 2011.

[8] G. Nicolis and I. Prigogine, "Exploring Complexities,” W. H. Freeman Co., New York, 1989.

[9] J. Skár, "Introduction: Self-Organization as an Actual Theme,” Philosophical Transactions of the Royal Society London, Vol. A 361, No. 1807, 2003, pp. 1049-1056.

[10] C. Chan, N. Herskowitz and K.-E. Longeen, "Electron Temperature Difference and Double Layers," Physics of Fluids, Vol. 26, No. 6, 1983, pp. 1585-1595. doi:10.1063/1.864294

[11] S. D. Baalrund, B. Longmier and N. Heshkowitz, "Equilibrium States of Anodic Double Layers," Plasma Sources Science and Technology, Vol. 18, No. 3, 2002, Article ID: 035002. doi:10.1088/0963-0252/18/3/035002

[12] T. Gyergyek, M. Cercek, R. Schrittwieswer and C. Ionita, "Experimental Study of the Creation of a Firerod by Langmuir and Emission Probes," Contributions to Plasma Physics, Vol. 42, No. 5, 2002, pp. 508-525. doi:10.1002/1521-3986(200208)42:5<508::AID-CTPP50

\section{8>3.0.CO;2-B}

[13] H. Fröhlich, "The Biological Effects of Microwaves and Related Questions," Advances in Electronics and Electron Physics, Vol. 53, 1980, pp. 85-152. doi:10.1016/S0065-2539(08)60259-0

[14] E. Lozneanu and M. Sanduloviciu, "Dynamical Self-Organization Acting as Physical Basis of the Ball Lightning Phenomenon,” In: M. D. Wood, Ed., Lightning: Proprieties, Formation and Types, Nova Science Publisher, Inc., New York, 2010.

[15] V. N. Tsytovich, et al., "From Plasma Crystals and Helical Structures toward Inorganic Living Matter," New Journal of Physics, Vol. 9, 2007, pp. 263-273. doi:10.1088/1367-2630/9/8/263

[16] D. Cohen, "Plasma Blobs Hint of New Form of Life," New Scientist, Vol. 179, No. 2413, 2003, pp. 16-17.

[17] L. M. Gabore, "Self-Other Organization: Why Early Life Did Not Evolves through Natural Selection,” Journal of Theoretical Biology, Vol. 241, No. 3, 2006, pp. 443-450.

[18] R. S. Hameroff and J. Tuszynski, "Quantum States in Protein and Protein Assembles: The Essence of Life," Proceedings of SPIE Conference on Fluctuations and Noise, Canary Islands, June 2004. 